\title{
The mnemonic advantage of processing fitness-relevant information
}

\author{
SeAn H. K. Kang, KathleEn B. MCDermott, AND Sophie M. Cohen \\ Washington University, St. Louis, Missouri
}

\begin{abstract}
Nairne, Thompson, and Pandeirada (2007) proposed that our memory systems serve an adaptive function and that they have evolved to help us remember fitness-relevant information. In a series of experiments, they demonstrated that processing words according to their survival relevance resulted in better retention than did rating them for pleasantness, personal relevance, or relevance to moving to a new house. The aim of the present study was to examine whether the advantage of survival processing could be replicated, using a control condition that was designed to match the survival processing task in arousal, novelty, and media exposure - the relevance to planning a bank heist. We found that survival processing nonetheless yielded better retention on both a recall (Experiment 1) and a recognition (Experiment 2) test. This mnemonic advantage of survival processing was also obtained when words were rated for their relevance to a character depicted in a video clip (Experiment 3). Our findings provide additional evidence that the mnemonic benefit of survival processing is a robust phenomenon, and they also support the utility of adopting a functional perspective in investigating memory.
\end{abstract}

Memory researchers have predominantly taken a structuralist approach to analyzing memory, preferring to focus on the proximate mechanisms that give rise to memory phenomena (Nairne, 2005). Studying the capabilities of human memory systems while ignoring their plausible functions risks impeding deeper understanding of the design of the systems and their relationships to other cognitive systems (Klein, Cosmides, Tooby, \& Chance, 2002). Although some amount of memory theorizing has explicitly considered the putative functions of memory from an evolutionary perspective (e.g., Glenberg, 1997; Sherry \& Schacter, 1987), functionally motivated empirical investigations of memory are relatively rare.

Recently, Nairne, Thompson, and Pandeirada (2007) used a functional-evolutionary approach to generate and test a hypothesis regarding memory performance. They suggested that our memory systems were selectively designed to process and retain fitness-relevant information because of the adaptive advantage to survival that such systems would confer. If this is the case, then processing information in terms of its survival value should have mnemonic consequences. In a series of experiments, Nairne et al. demonstrated that words incidentally processed for their relevance to a survival scenario were especially well remembered. This advantage of survival processing was found in both between- and withinsubjects designs, as well as when memory was assessed by recall and recognition. The main control condition used in their study (in three out of four experiments) was a processing scenario involving relevance to moving to a new house.

Although this moving condition was designed to induce schema activation and meaningful processing (as would be expected in the survival condition), we felt that the moving scenario was somewhat mundane (cf. being stranded in the grassland and having to plan for one's survival), leaving open the challenge that the obtained memorial advantage of survival processing was perhaps due to that condition's being more novel and exciting. The aim of the present study was to investigate whether Nairne et al.'s (2007) findings were replicable under a control condition designed with this concern in mind, to test the robustness of the phenomenon. Specifically, we constructed a burglary scenario in which subjects imagined planning a bank heist and rated words according to their relevance to this scenario. The burglary scenario was created with the goal of being more equivalent to the survival scenario on qualities like excitement and novelty. Moreover, another aspect that both scenarios have in common is that they have been featured prominently in the central themes of several movies and television programs (e.g., bank heist, Inside Man, The Nine; survival, Cast Away, Survivor). Since our goal was to attempt a replication of the results of Nairne et al., we closely modeled the procedure of our first two experiments on theirs.

In Experiment 1, using a within-subjects design, we compared the effect of rating words for their survival relevance, their relevance to planning a burglary, and their pleasantness (considered one of the best semantic processing tasks; Packman \& Battig, 1978) on subsequent recall. In their first experiment, Nairne et al. (2007) also used pleasantness ratings as a second control condition (in addition to the moving control condition), but they used a between-subjects design. Their second experiment

S. H. K. Kang, seankang@wustl.edu 
switched to a within-subjects design, but they dropped the pleasantness rating condition. In Experiment 2, we sought to replicate the pattern of results with a recognition test, again using a within-subjects design (similar to Nairne et al.'s [2007] third experiment). In Experiment 3, we tested the generalizability of the survival processing advantage by modifying the encoding manipulation. Instead of imagining themselves in particular scenarios and rating words for their relevance, subjects watched video clips taken from movies and then rated words for their relevance to the characters portrayed in the video.

\section{EXPERIMENT 1}

\section{Method}

Subjects. Forty-eight undergraduates from the Washington University psychology subject pool participated in partial fulfillment of course requirements.

Materials. The stimuli consisted of 48 words - each being a typical exemplar from a unique category from the updated Battig and Montague norms (Van Overschelde, Rawson, \& Dunlosky, 2004) - divided into three sets matched on frequency $(M=48$; Kučera \& Francis, 1967), length $(M=5.5$ letters $)$, and imageability $(M=577$; Coltheart, 1981).

Design. A within-subjects design was used. Subjects rated each set of 16 words according to one of the three rating scenarios, and the rating task was blocked by scenario. The words in each set were presented in a random order, but the order of the three sets of words was fixed. The order of the rating scenarios was fully counterbalanced across subjects. Immediately after the rating task, subjects performed a distractor task for $5 \mathrm{~min}$, followed by a free-recall test of all rated words.

Procedure. Subjects were seated at computer terminals, and they were told that the experiment involved rating words according to their relevance to particular scenarios. The instructions for each of the three scenarios were:

Survival. In this task we would like you to imagine that you are stranded in the grasslands of a foreign land, without any basic survival materials. Over the next few months, you'll need to find steady supplies of food and water and protect yourself from predators. We are going to show you a list of words, and we would like you to rate how relevant each of these words would be for you in this survival situation. Some of the words may be relevant and others may not-it's up to you to decide.

Burglary. In this task we would like you to imagine that you are leading a heist of a well guarded bank. Over the next few months, you'll need to find people to help you, make a plan, and gather any supplies you might need. We are going to show you a list of words, and we would like you to rate how relevant each of these words would be for you in accomplishing this task. Some of the words may be relevant and others may notit's up to you to decide. ${ }^{1}$

Pleasantness. In this task, we are going to show you a list of words, and we would like you to rate the pleasantness of each word. Some of the words may be pleasant and others may notit's up to you to decide.

The word stimuli were presented one at a time in a central location on the screen for $5 \mathrm{sec}$ each, and subjects were asked to rate them on a 5 -point scale (i.e., $1=$ totally irrelevant/unpleasant; $5=$ totally relevant/pleasant). The rating scale was presented below each stimulus, and subjects made their ratings by pressing the appropriate number key on the keyboard. Subjects were instructed to respond within the $5 \mathrm{sec}$ that each word was up on the screen. They were not informed of the upcoming memory test.

Following the word rating task, subjects played a Tetris video game for $5 \mathrm{~min}$, after which they were interrupted and asked to recall as many of the earlier rated words as they could. The free recall test lasted for $10 \mathrm{~min}$, and subjects were instructed to type in their responses in any order they wished. At the end of the experiment, subjects were debriefed and thanked for their participation.

\section{Results and Discussion}

Recall. The left panel of Figure 1 shows the mean proportion of words recalled for the three rating conditions. As can be seen from the figure, rating words according to their relevance to survival produced better retention than did rating words for their relevance to a burglary or for their pleasantness. This result was confirmed by statistical tests $(\alpha$ level for all statistical analyses was set at $p<$ $.05)$, which revealed a significant effect of rating condition $\left[F(2,94)=9.219, M S_{\mathrm{e}}=.022, \eta_{\mathrm{p}}^{2}=.164\right]$. Recall for the survival condition was significantly higher than that for both the burglary and pleasantness conditions $[t(47)=$ $2.57, d=.37$, and $t(47)=4.75, d=.69$, respectively]. Performance for the burglary and pleasantness conditions did not differ $[t(47)=1.47]$.

Rating. The middle panel of Figure 1 shows the mean ratings given to the words for each condition. The highest ratings were obtained in the pleasantness condition; the lowest were in the burglary condition, and the survival condition was in the middle. There was a significant effect of rating condition $\left[F(2,94)=73.566, M S_{\mathrm{e}}=.134, \eta_{\mathrm{p}}^{2}=\right.$ .610]. The pleasantness condition yielded significantly higher ratings than did the survival condition $[t(47)=$
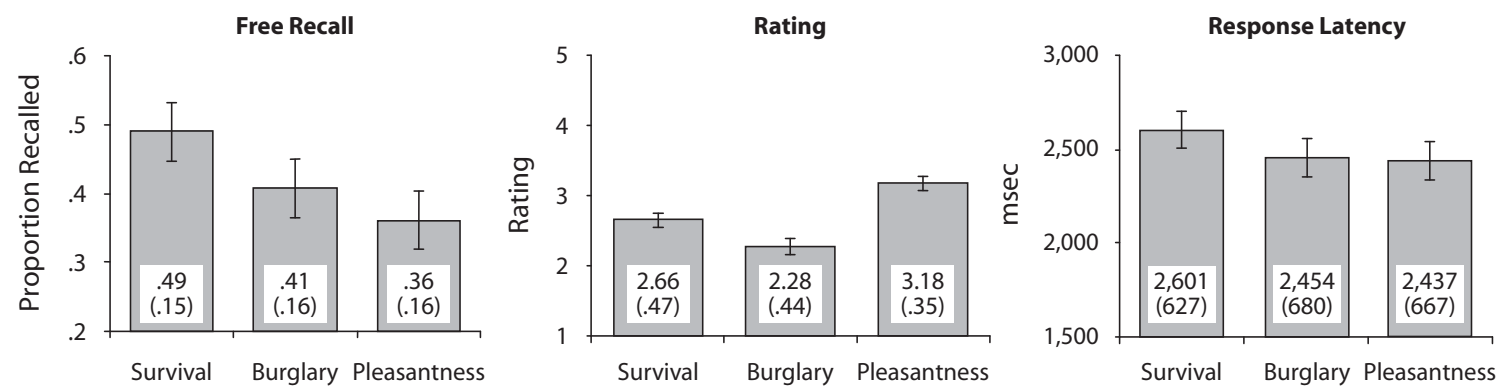

Figure 1. Mean proportion of words recalled, mean rating, and mean response latency during rating task as a function of condition for Experiment 1. Error bars are $\mathbf{9 5 \%}$ confidence intervals. Means and standard deviations (in parentheses) for each condition are listed in the respective bars. 
$6.22, d=.90]$, which in turn yielded higher ratings than did the burglary condition $[t(47)=5.12, d=.74]$.

Response latency. The right panel of Figure 1 shows the mean response time (RT) during the rating task for each condition. Subjects took a longer time to rate words for the survival condition than for the other two conditions. There was a significant effect of rating condition $[F(2,94)=$ $\left.3.305, M S_{\mathrm{e}}=119,080.980, \eta_{\mathrm{p}}^{2}=.066\right]$. Responses in the survival condition were slower than in the pleasantness condition $[t(47)=2.61, d=.38]$ and in the burglary condition $[t(47)=2.09, d=.38]$. There was no difference between the burglary and pleasantness conditions $[t(47)<1]$.

In summary, processing words according to their relevance to one's survival led to better retention, even when compared with a scenario that was designed to closely match the survival scenario in terms of novelty, excitement value, and media portrayal. In fact, processing words according to how relevant they might be to planning a bank heist provided no mnemonic benefit over the prototypical "deep" processing task (i.e., pleasantness ratings). The rating data do not rule out the possibility that the advantage of survival processing was mediated by a congruity effect (Schulman, 1974); that is, items rated higher (more relevant) are better integrated with the processing scenario, and, hence, they are more likely to be subsequently retrieved. The survival condition yielded higher relevance ratings than did the burglary condition, and it is possible that the greater congruity between the items and the survival scenario was at least partly responsible for the mnemonic advantage. Although ratings for the pleasantness condition were the highest, it could be argued that pleasantness ratings do not reflect the degree of integration between items and an overarching schema (as would be the case when items were rated for their relevance to a scenario). Also, since the survival rating task took more time than the pleasantness and burglary rating tasks, one could argue that the better performance in the survival condition was due to more effortful or deeper processing of the words at encoding (Craik \& Lockhart, 1972). It should be mentioned, however, that processing time during similar rating tasks has been found to be unrelated to subsequent memory performance (see, e.g., Craik \& Tulving, 1975). Nonetheless, we felt it was important to replicate our findings with a different set of stimuli and to ascertain whether the obtained pattern of memory performance would generalize to a different retrieval task.

\section{EXPERIMENT 2}

The previous experiment found that the recall advantage of survival processing persisted, even when compared with a control condition designed to contain elements of emotional arousal and novelty. Experiment 2 was designed to examine whether this phenomenon extended to recognition memory. Again, a within-subjects design was used, but we decided to drop the pleasantness rating condition and just compare the survival condition with the burglary condition. Also, a free-choice (old-new) recognition test was administered instead of a recall test.

\section{Method}

Subjects. Forty-eight undergraduates from the Washington University psychology subject pool participated in partial fulfillment of course requirements. These subjects did not participate in the previous experiment.

Materials. The stimuli consisted of 128 words selected from the Clark and Paivio (2004) norms, divided into four sets matched on frequency $(M=226)$, length $(M=5.75)$, imageability $(M=527)$, and familiarity $(M=578$; Coltheart, 1981).

Design and Procedure. A within-subjects design was used. Subjects rated 32 words using the survival scenario (S) and 32 words using the burglary scenario (B), with the rating condition blocked in trials of 16 words (SBSB or BSBS, counterbalanced across subjects). The four sets of words each served equally in each rating condition and as distractors in the recognition test. The words in each set were presented in a random order. The procedure was identical to that in Experiment 1, except that the rating conditions alternated in blocks of 16 trials (with scenario instructions presented at the start of each block). In addition, the duration of the distractor task (Tetris video game) was lengthened to $10 \mathrm{~min}$, and this was followed by a self-paced, free-choice recognition test. Subjects were presented, one at a time, 128 words (64 words rated earlier, 64 distractors) and were asked to indicate with a keypress whether each word was "old" (i.e., rated earlier) or "new" (i.e., not rated earlier). The target and distractor words in the test were randomly ordered.

\section{Results and Discussion}

Recognition. The left panel of Figure 2 shows the mean proportion of target items correctly classified as "old" for the survival and burglary conditions. The mean overall false alarm rate (i.e., distractor items classified as "old")
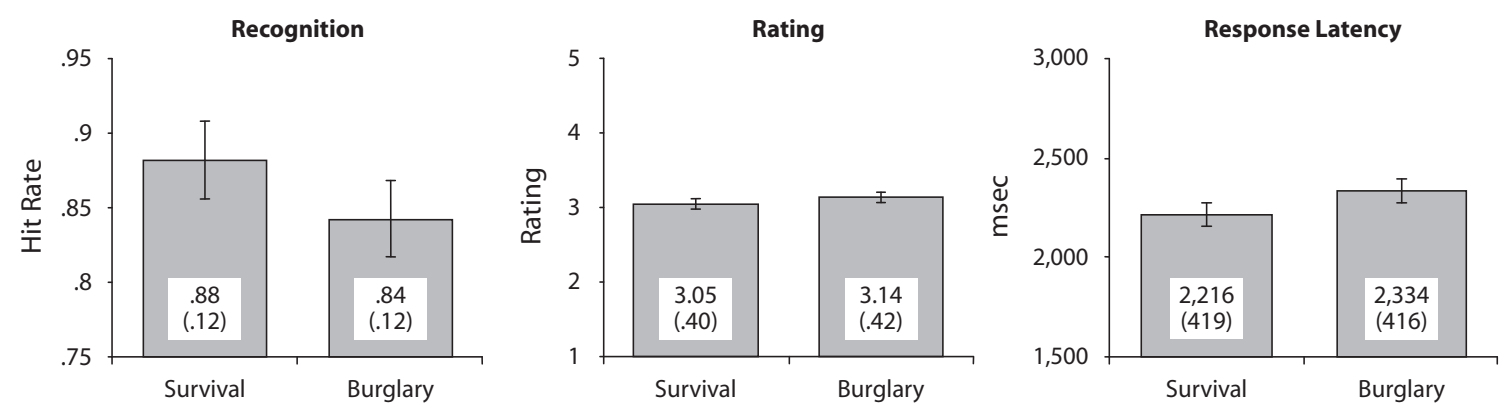

Figure 2. Mean recognition hit rate, mean rating, and mean response latency during rating task as a function of condition for Experiment 2. Error bars are $\mathbf{9 5 \%}$ confidence intervals. Means and standard deviations (in parentheses) for each condition are listed in the respective bars. 
was .15. As can be seen from the figure, recognition performance was higher for the survival condition than for the burglary condition $[t(47)=2.14, d=.31]$. Of the 48 subjects, 30 correctly recognized more words for the survival than for the burglary condition; 13 showed the opposite pattern, and 5 were tied.

Rating. The middle panel of Figure 2 shows the mean ratings of words for the survival and burglary conditions. Due to a computer error, the rating data for 6 subjects were lost; thus, the analyses of ratings and their latencies were based on data from the remaining 42 subjects. With the different set of stimuli used in Experiment 2, the words were rated as marginally more relevant for the burglary condition than for the survival condition (cf. Experiment 1 , where the opposite result was obtained) $[t(41)=$ $1.79, p=.081, d=.28]$.

Response latency. The right panel of Figure 2 shows the mean amount of time it took for subjects to rate the words for the survival and burglary conditions. In Experiment 1 , subjects took slightly more time to rate words for the survival than for the burglary condition, whereas in the present experiment, more time was taken during the rating task for the burglary condition than for the survival condition $[t(41)=2.82, d=.44]$.

The primary results of Experiment 2 are the replication of the previous finding of a mnemonic advantage of survival processing and the extension to recognition memory. This benefit of processing items for their relevance to survival was neither due to a greater expense of time during the rating task (rating responses for the burglary condition took longer) nor to a greater congruity between the target items and the processing scenario (words were judged to be slightly more relevant for the burglary condition).

\section{EXPERIMENT 3}

The results from the previous two experiments confirm the memorial advantage of survival processing, at least when words are processed for their relevance to one's survival. In the procedure we used thus far (adapted from Nairne et al., 2007), survival processing was induced by asking subjects to imagine themselves in a scenario that required them to plan for their survival. Hence, the tobe-remembered words were processed for their relevance to the survival of the self. Although Nairne et al. (2007, Experiment 4) demonstrated that the survival processing advantage was not merely due to the self-reference effect (i.e., the mnemonic benefit produced when information is related to the self; Symons \& Johnson, 1997), it was unclear whether the concept of the self was necessary for the survival advantage to be instantiated.

The aim of Experiment 3 was twofold: to ascertain whether the advantage of survival processing would persist when the concept of the self was removed from the scenario, and in so doing, to determine the generalizability of the survival processing manipulation beyond what Nairne et al. (2007) used. Instead of asking subjects to read about and envision themselves in a survival scenario, they watched a short video clip consisting of scenes related to survival on a deserted island, and then they rated words for their relevance to the portrayed character's survival.

\section{Method}

Subjects. Forty-eight undergraduates from the Washington University psychology subject pool participated in partial fulfillment of course requirements. These subjects did not participate in the previous two experiments.

Materials. The stimuli consisted of 42 words selected from the Clark and Paivio (2004) norms, divided into three sets matched on frequency $(M=49)$, length $(M=5.7)$, imageability $(M=597)$, and familiarity $(M=547)$. The video clip used in the survival condition was taken from the movie Cast Away, and it consisted of scenes of the main character being in an airplane that crashes into the sea; subsequently, he washes up onto a deserted island. The video clip used in the burglary condition was taken from the movie Inside Man, and it consisted of scenes of a bank heist being executed, followed by the main character talking about his motives for robbing the bank. Both clips were about $90 \mathrm{sec}$ in length and contained audio.

Design. A within-subjects design was used. Subjects rated each set of 14 words according to one of the three rating scenarios (i.e., survival, burglary, and pleasantness), and the rating task was blocked by scenario. The words in each set were presented in a random order, but the order of the three sets of words was fixed. The order of the rating scenarios was fully counterbalanced across subjects. Immediately after the rating task, subjects performed a distractor task for 5 min, followed by a free recall test of all rated words.

Procedure. Subjects were seated at computer terminals and were told that the experiment involved rating words according to their relevance to particular scenarios. For the survival and burglary conditions, subjects were asked to watch the appropriate video clip, after which the following instructions were given:

You've just watched a short video clip of a man being stranded on a deserted island (or involved in a bank robbery). Next, you will be presented with a list of words. Your task is to rate how relevant each of these words might be for the character in the video clip in terms of planning for his survival (or planning his bank heist). Some of the words may be relevant and others may not-it's up to you to decide.

The instructions for the pleasantness condition and the rest of the procedure (e.g., presentation rate for words, rating scale, recall test, etc.) were identical to those used in Experiment 1. In addition, subjects were asked at the end of the experiment whether they had previously watched the movies Cast Away and Inside Man.

\section{Results and Discussion}

Recall. The left panel of Figure 3 shows the mean proportion of words recalled for the three rating conditions. As can be seen from the figure, once again, rating words according to their relevance to survival produced better retention than did rating words for their relevance to a burglary or for their pleasantness. This result was confirmed by statistical tests, which revealed a significant effect of rating condition $\left[F(2,94)=6.433, M S_{\mathrm{e}}=.022, \eta_{\mathrm{p}}^{2}=.12\right]$. Recall for the survival condition was significantly higher than that for both the burglary and the pleasantness conditions $[t(47)=2.23, d=.32$, and $t(47)=3.77, d=.54$, respectively]. Performance for the burglary and pleasantness conditions did not differ $[t(47)=1.14]$.

Rating. The middle panel of Figure 3 shows the mean ratings given to the words for each condition. The highest ratings were obtained in the pleasantness condition, the lowest in the burglary condition, and the survival condi- 

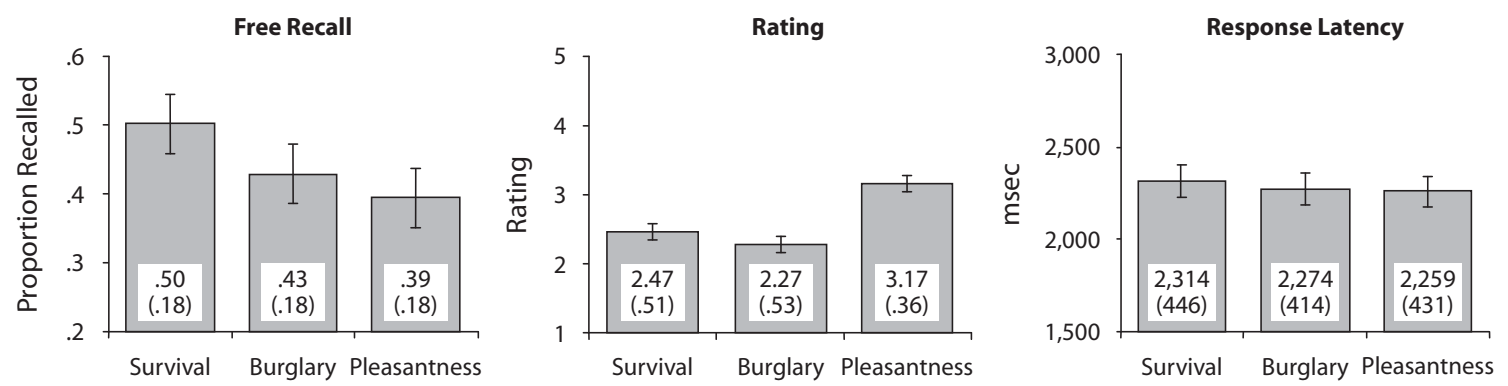

Figure 3. Mean proportion of words recalled, mean rating, and mean response latency during rating task as a function of condition for Experiment 3. Error bars are $\mathbf{9 5 \%}$ confidence intervals. Means and standard deviations (in parentheses) for each condition are listed in the respective bars.

tion was in the middle. There was a significant effect of rating condition $\left[F(2,94)=64.408, M S_{\mathrm{e}}=.166, \eta_{\mathrm{p}}^{2}=\right.$ $.578]$. The pleasantness condition yielded significantly higher ratings than did the survival condition $[t(47)=$ $8.40, d=1.21]$, which in turn yielded higher ratings than did the burglary condition $[t(47)=2.37, d=.34]$.

Response latency. The right panel of Figure 3 shows the mean RT during the rating task for each condition. The RTs did not differ across the three conditions $(F<1)$.

In summary, Experiment 3 replicated the previous findings of a mnemonic advantage of survival processing. Importantly, this advantage was not contingent on processing words for relevance to the survival of one's self. The benefit was obtained even when subjects considered the words for relevance to the survival of a fictitious character in a movie. Also, the survival advantage cannot be attributed to differences in processing time. In addition, whether subjects had previously viewed the movies from which the video clips were taken did not affect performance during the rating task nor at recall [there was no interaction with rating condition when having formerly viewed the movies was included as a between-subjects factor in the analyses $(F<1)]$.

\section{GENERAL DISCUSSION}

The results of the present experiments provide further evidence that processing items for their relevance to survival enhances retention of those items. More specifically, our study showed that this memorial benefit was found regardless of whether memory was assessed with recall (Experiment 1) or recognition (Experiment 2), and that the advantage could be attributed neither to differences in processing time during the orienting task nor to greater congruity between target items and the processing scenario. The survival processing task still retained its superiority when pitted against a rival scenario that was specifically designed to match it on various dimensions. The main comparisons in Nairne et al. (2007) were between survival processing and a moving scenario. Using these comparisons left open the possible criticism that perhaps the moving condition was not as unusual or thrilling from the subject's point of view; hence, the survival advantage could potentially be explained away in terms of the survival scenario providing more distinctive processing (Hunt \& McDaniel, 1993) or arousal. However, our findings help rule out this alternative explanation and provide additional confirmation that the memorial benefit of survival processing is a robust phenomenon.

Additionally, for survival processing to boost retention, we found that it was unnecessary for the processing to involve survival of the self; the same effect was found when items were processed for their relevance to another person's survival (Experiment 3). Perhaps this is not too surprising, given the human capacity for perspective taking (see, e.g., Decety \& Sommerville, 2003). Nonetheless, our findings suggest that an explicit contemplation of one's own survival may not be critical to the phenomenon and that the same benefit can be achieved when considering the survival of others.

Our study was not aimed at determining the exact proximate mechanisms that give rise to the memorial benefit of survival processing. Aside from suggesting that this advantage cannot be ascribed solely to greater distinctiveness or arousal, anything more would be pure conjecture. Nairne et al. (2007) speculated that one possibility could be that survival processing draws on special cognitive adaptations or modules that have been designed to solve specific problems in our ancestral past (e.g., foraging for food, avoidance of predators/contaminants). Specifying how these putative modules are involved in survival processing will depend on future research (a challenging endeavor, given the debate about the identity of these modules; see Buller, 2005).

In conclusion, the mnemonic benefit of survival processing appears to be a robust phenomenon. This finding is consistent with the idea that our memory systems developed to process and remember fitness-relevant information, and it further validates the functional-evolutionary approach to studying memory.

\section{AUTHOR NOTE}

Parts of this study were presented in a poster at the 48th Annual Meeting of the Psychonomic Society, Long Beach, CA, in November 2007. The authors acknowledge H. L. Roediger III for his helpful comments. Correspondence concerning this article should be addressed to S. H. K. Kang, Department of Psychology, Washington University, Saint Louis, MO 63130-4899 (e-mail: seankang@wustl.edu). 


\section{REFERENCES}

Buller, D. J. (2005). Evolutionary psychology: The emperor's new paradigm. Trends in Cognitive Sciences, 9, 277-283.

Clark, J. M., \& Paivio, A. (2004). Extensions of the Paivio, Yuille, and Madigan (1968) norms. Behavior Research Methods, Instruments, \& Computers, 36, 371-383.

Coltheart, M. (1981). The MRC psycholinguistic database. Quarterly Journal of Experimental Psychology, 33A, 497-505.

Craik, F. I. M., \& LockharT, R. S. (1972). Levels of processing: A framework for memory research. Journal of Verbal Learning \& Verbal Behavior, 11, 671-684.

Craik, F. I. M., \& Tulving, E. (1975). Depth of processing and the retention of words in episodic memory. Journal of Experimental Psychology: General, 104, 268-294.

Decety, J., \& Sommerville, J. A. (2003). Shared representations between self and other: A social cognitive neuroscience view. Trends in Cognitive Sciences, 7, 527-533.

Glenberg, A. M. (1997). What memory is for. Behavioral \& Brain Sciences, 20, 1-55.

Hunt, R. R., \& McDaniel, M. A. (1993). The enigma of organization and distinctiveness. Journal of Memory \& Language, 32, 421-445.

Klein, S. B., Cosmides, L., Tooby, J., \& Chance, S. (2002). Decisions and the evolution of memory: Multiple systems, multiple functions. Psychological Review, 109, 306-329.

KuČERA, H., \& FRANCIS, W. (1967). Computational analysis of presentday American English. Providence, RI: Brown University Press.

NAIRNE, J. S. (2005). The functionalist agenda in memory research. In A. F. Healy (Ed.), Experimental psychology and its applications (pp. 115126). Washington, DC: American Psychological Association.

Nairne, J. S., Thompson, S. R., \& Pandeirada, J. N. S. (2007). Adaptive memory: Survival processing enhances retention. Journal of Experimental Psychology: Learning, Memory, \& Cognition, 33, 263-273.

Packman, J. L., \& Battig, W. F. (1978). Effects of different kinds of semantic processing on memory for words. Memory \& Cognition, 6, 502-508.

Schulman, A. I. (1974). Memory for words recently classified. Memory \& Cognition, 2, 47-52.

Sherry, D. F., \& Schacter, D. L. (1987). The evolution of multiple memory systems. Psychological Review, 94, 439-454.

Symons, C. S., \& Johnson, B. T. (1997). The self-reference effect in memory: A meta-analysis. Psychological Bulletin, 121, 371-394.

Van Overschelde, J. P., Rawson, K. A., \& Dunlosky, J. (2004). Category norms: An updated and expanded version of the Battig and Montague (1969) norms. Journal of Memory \& Language, 50, 289-335.

\section{NOTE}

1. Normative ratings of the survival and burglary scenarios were collected. Subjects were presented with both scenarios (the order was counterbalanced; $N=30$ ) and were asked to rate them-using 5-point Likert scales - on excitement (How exciting would you consider the scenario? 1 = very unexciting; 5 = very exciting), novelty (How often do you imagine yourself in such a scenario? $1=$ never; 5 = very often), and media exposure (How often do you encounter such a scenario in the media? $1=$ never; 5 = very often $)$. The scenarios did not differ on excitement (survival $M=3.57$; burglary $M=3.93$ ) $[t(29)=1.266]$ or on novelty (survival $M=1.90$; burglary $M=1.67$ ) $[t(29)=1.045]$. The burglary scenario, however, was rated as being encountered more often in the media than the survival scenario ( $M \mathrm{~s}=3.53$ and 3.13 , respectively) $[t(29)=2.693]$. Nonetheless, the burglary scenario served as an appropriate control condition because any memory advantage yielded by survival processing could not be due to the survival scenario's having greater media exposure (or being more exciting or novel).

(Manuscript received October 29, 2007; revision accepted for publication March 20, 2008.) 